\title{
Small molecules, inhibitors of DNA-PK, targeting DNA repair, and beyond
}

\section{David Davidson, Lilian Amrein, Lawrence Panasci * and Raquel Aloyz *}

Department of Oncology, Segal Cancer Centre, Lady Davis Institute for Medical Research, Jewish General Hospital, McGill University, Montreal, OC, Canada

\section{Edited by:}

Gerald Batist, McGill University,

Canada

\section{Reviewed by:}

Rayudu Gopalakrishna, University of

Southern California, USA

Jian Hui Wu, McGill University,

Canada

\section{*Correspondence:}

Lawrence Panasci and Raquel Aloyz, Oncology, Segal Cancer Centre, Lady Davis Institute, Jewish General Hospital, 3755 Côte St Catherine, Montreal, OC, H3T 1E2, Canada. e-mail: Ipanasci@hotmail.com; raquel.aloyz@mcgill.ca
Many current chemotherapies function by damaging genomic DNA in rapidly dividing cells ultimately leading to cell death. This therapeutic approach differentially targets cancer cells that generally display rapid cell division compared to normal tissue cells. However, although these treatments are initially effective in arresting tumor growth and reducing tumor burden, resistance and disease progression eventually occur. A major mechanism underlying this resistance is increased levels of cellular DNA repair. Most cells have complex mechanisms in place to repair DNA damage that occurs due to environmental exposures or normal metabolic processes. These systems, initially overwhelmed when faced with chemotherapy induced DNA damage, become more efficient under constant selective pressure and as a result chemotherapies become less effective. Thus, inhibiting DNA repair pathways using target specific small molecule inhibitors may overcome cellular resistance to DNA damaging chemotherapies. Non-homologous end joining a major mechanism for the repair of double-strand breaks (DSB) in DNA is regulated in part by the serine/threonine kinase, DNA dependent protein kinase (DNA-PK). The DNA-PK holoenzyme acts as a scaffold protein tethering broken DNA ends and recruiting other repair molecules. It also has enzymatic activity that may be involved in DNA damage signaling. Because of its' central role in repair of DSBs, DNA-PK has been the focus of a number of small molecule studies. In these studies specific DNA-PK inhibitors have shown efficacy in synergizing chemotherapies in vitro. However, compounds currently known to specifically inhibit DNA-PK are limited by poor pharmacokinetics: these compounds have poor solubility and have high metabolic lability in vivo leading to short serum half-lives. Future improvement in DNA-PK inhibition will likely be achieved by designing new molecules based on the recently reported crystallographic structure of DNA-PK. Computer based drug design will not only assist in identifying novel functional moieties to replace the metabolically labile morpholino group but will also facilitate the design of molecules to target the DNA-PKcs/Ku80 interface or one of the autophosphorylation sites.

Keywords: DNA-PK, DNA-PKcs, DNA damage, DNA repair, DNA-PK inhibitors

\section{INTRODUCTION}

Many first-line cancer therapies employ DNA damaging chemotherapeutic agents alone or in combination with ionizing radiation (IR; Seymour et al., 2007; Cardoso et al., 2009; Tol et al., 2009; Raftery and Goldberg, 2010). These therapies primarily target genomic DNA resulting in the formation of highly toxic double-strand DNA breaks (DSBs) leading to cell cycle arrest and cell death. Problematic to cancer treatment, cells have multiple mechanisms for repairing DSBs including homologous recombination (HR; Johnson and Jasin, 2001; Kao et al., 2005; Sung and Klein, 2006) and non-homologous end joining (NHEJ; Burma et al., 2006; Lieber et al., 2006; Bennardo et al., 2008; Lieber, 2008). $\mathrm{HR}$, an error free repair process, depends on the availability of a homologous DNA template and, as such, functions primarily in the G2/M phase of the cell cycle when sister chromatids are in close proximity. NHEJ, although error prone compared to HR, is thought to be the main pathway for the repair of DNA DSBs. This pathway is able to function at all stages of the cell cycle and does not require the presence of a homologous template. The risk of mutation to an essential gene as a result of NHEJ is balanced by the benefit of avoiding catastrophic cell division in the presence of DSBs. As such, it has been hypothesized that targeting the molecular machinery driving the DNA damage response (DDR), particularly NHEJ and DSB repair, with small molecule inhibitors, will effectively enhance the efficacy of current cancer treatments that generate DNA damage.

\section{DNA DEPENDENT PROTEIN KINASE}

One such target, DNA dependent protein kinase (DNA-PK), a nuclear serine/threonine kinase, is a critical protein facilitating NHEJ (Durocher and Jackson, 2001; Yang et al., 2003, 2009; Collis et al., 2005; Dobbs et al., 2010; Neal and Meek, 2011; Neal et al., 2011). The DNA-PK holoenzyme is composed a regulatory heterodimer (Ku70 and Ku80 subunits) and a $460 \mathrm{kD}$ catalytic component, DNA-PKcs. Based on amino acid sequence homology DNA-PK is a member of the phosphatidylinositol-3-kinase 
superfamily, however, since it possesses no apparent lipid kinase activity it has been classified along with Ataxia Telangiectasia Mutated (ATM), Ataxia Telangiectasia and Rad3 Related (ATR), Mammalian Target of Rapamycin (m-Tor), and Suppressor of Morphogenesis in Genitalia-1 (SMG-1) as part of the phosphatidyl inositol 3-kinase-like (PIKK) protein kinase group (Durocher and Jackson, 2001). Along with ATM and ATR, DNA-PK has a central function in the DSB response and collectively these three kinases are capable of phosphorylating upwards of 700 proteins (in vitro; Callen et al., 2009). DNA-PK itself is a target of ATM and ATR and is essential to NHEJ, V(D)J recombination and class switch recombination (Ma et al., 2002; Callen et al., 2009; Neal and Meek, 2011), all of which require the repair of DSBs. In vitro DNA-PKcs is activated by free DNA ends and is capable of phosphorylating several protein substrates including the $\mathrm{Ku} 70 / 80$ heterodimer, histone variant $\mathrm{H} 2 \mathrm{AX}$, replication associated protein A (RPA), and autophosphorylation (Stiff et al., 2004; Reitsema et al., 2005; Wang et al., 2005; Mukherjee et al., 2006; Koike et al., 2008; An et al., 2010). Furthermore, DNA-PK depended phosphorylation of S473 of AKT in response to platinum based chemotherapy has been shown to inhibit apoptotic response limiting drug efficacy (Stronack et al., 2011). There is also in vitro evidence that DNA-PK interacts with or influences $\mathrm{p} 53$ and p21 activities leading to cellular senescence and apoptosis (Azad et al., 2011; Rudolf et al., 2011; Stronack et al., 2011; Wei et al., 2012). Even so, only a few in vivo DNA-PK substrates are known, these include DNA-PKcs autophosphorylation and histone variant H2AX. H2AX phosphorylation on S139 $(\gamma \mathrm{H} 2 \mathrm{AX})$ has been linked to Akt activity downstream of DNA-PK with S473 of Akt being phosphorylated by DNA-PK. In response to this phosphorylation GSK3 $\beta$, a negative regulator of DNA-PK, is phosphorylated on Ser9 which prevents GSK3 $\beta$ from dephosphorylating $\gamma \mathrm{H} 2 \mathrm{AX}$ (An et al., 2010). Despite the small number of substrates identified in vivo DNA-PK kinase activity is essential to efficient DSB repair as only wild type and not kinase dead enzyme can rescue IR sensitivity in DNA-PKcs defective V3 cells (Chen et al., 2005). Furthermore, cells displaying resistance to DNA damaging agents had increased levels of DNA-PKcs activity and cells deficient in DNA-PKcs had enhanced sensitivity to DNA damaging agents (Soubeyrand et al., 2003; Shinohara et al., 2005; Morris et al., 2011). Also, although phosphorylation of DNA-PK and DNA-PK kinase activity are not required for initial recruitment to DNA damage sites they are required for efficient repair of DSBs (Davis et al., 2010). It has been proposed that DNA-PK functions as a scaffolding protein to align the broken DNA ends and assist in the localization of repair factors. It may also play a regulatory role by physically blocking unrepaired DNA ends preventing their degradation, then, through autophosphorylation, releasing them for repair once proper alignment has been achieved (Neal and Meek, 2011; Neal et al., 2011).

Of note, a recent review by Kong et al. (2011) discusses physiological functions of DNA-PK beyond its' role in DNA repair.

These authors note that the abundant expression of the large DNA-PK protein molecule in numerous cell types does not impart improved DNA repair abilities, suggesting other important functions for this molecule may exist. Evidence presented suggests that DNA-PK has important roles in regulating gene response to feeding/insulin stimulation. Furthermore, it is suggested that DNA-PK has an active part in the regulation of homeostasis of cell proliferation. Given that cancer cells generally have a high rate of proliferation and have a high rate of metabolic activity these additional functions suggest further mechanisms by which DNA-PK inhibition can target cancer cells.

\section{MECHANISM}

Regarding the role of DNA-PK in DNA repair a recent model of NHEJ was proposed by Neal and Meek (2011) that suggests DSB repair is initiated when the regulatory component of DNA-PK, $\mathrm{Ku} 70 / \mathrm{Ku} 80$, comes in contact with DNA ends at DSB sites. The $\mathrm{Ku}$ heterodimer, which is able to bind to various double-stranded end structures (blunt ends, $3^{\prime}$ or $5^{\prime}$ overhangs, covalently closed hairpins) in a sequence independent manner, forms an asymmetric ring that encircles double-stranded DNA ends. Subsequently, DNA-PKcs is recruited and binds to the C-terminal portion of Ku80 (Morris et al., 2011). DNA bound $\mathrm{Ku}$ then translocates inwards away from the broken end significantly stimulating the kinase activity of DNA-PKcs. It is proposed that homo-dimeric interaction of DNA bound DNA-PK complexes brings corresponding broken ends into close proximity and subsequently acts as a scaffold for recruiting downstream repair proteins such as XRCC4, DNA ligase IV, Artemis, XLF, and aprataxin. Activity of this larger protein complex ultimately results in the repair of DSBs (Kuo et al., 2006; Mansour et al., 2008). Molecular models of DNA-PK associated DSB repair have been reviewed in detail by Thompson (2012). The initial step of DSB repair occurs rapidly with both ATM and DNA-PK being recruited to DNA damage sites. DNA-PK is recruited within $60 \mathrm{~s}$ and recruitment peaks after $10 \mathrm{~min}$. In contrast ATM recruitment peaks after $20 \mathrm{~min}$. DNA-PK rapidly dissociates after reaching peak levels and in contrast ATM lingers for a prolonged period. Dissociation of DNA-PK, ATM, and other DNA repair factors from DSB sites is associated with the resolution of $\gamma \mathrm{H} 2 \mathrm{AX}$ foci suggesting successful DSB repair. In contrast, DNA-PK showed persistent localization in NHEJ deficient cells (Uematsu et al., 2007). These data suggest a model where DNA-PK and ATM hold separate and distinct functions in DSB repair with DNA-PK functioning as a scaffold and ATM acting as a signaling kinase.

\section{PHOSPHORYLATION}

DNA dependent protein kinase is clearly important for efficient repair of DSBs and to date as many as 40 potential phosphorylation sites have been identified on DNA-PK with the majority of these being located in two major clusters: (1) the ABCDE cluster containing S2612, S2624, T2609, T2620, T2638, T2647 and 2) the PQR cluster containing sites S2023, S2029, S2041, S2053, and S2056 (Morris et al., 2011; Neal and Meek, 2011; Neal et al., 2011). Mechanistically, experimental evidence suggests that unphosphorylated DNA-PK blocks DNA ends inhibiting efficient ligation and that autophosphorylation generates a large conformational change opening the structure of DNA-PK (Davis et al., 2010; Morris et al., 2011) releasing it from aligned DNA ends. For example, cells expressing mutations at S2056 or T2609 have tight binding of DNA-PK to DNA DSBs and a slow off rate at sites of DNA 
damage (Davis et al., 2010). The structure of the DNA-PKcs molecule has three major domains facilitating this functionality: a kinase domain, a large $\mathrm{N}$-terminal domain that includes two long arms into which DNA is proposed to thread and a DNA binding pocket. The phosphorylations of amino acids from the respective clusters, S2056 (PQR) and T2609 (ABCDE), are likely distinctly regulated events as the physical locations of these residues are far apart (Chen et al., 2005). Furthermore, phosphorylations of these clusters may be functionally distinct in that ABCDE and PQR clusters were shown to regulate DNA end processing and DNA repair pathway choice in a reciprocal manner. For example, blocking at the amino acid cluster ABCDE inhibited end processing and HR while blocking at the PQR cluster enhanced these processes. Additionally, it was observed that S2056 phosphorylation increased incrementally and reached maximum levels at $50 \mathrm{~Gy}$ of IR. This was in contrast to T2609 whose phosphorylation reached maximum levels at $10 \mathrm{~Gy}$. Based on the use of ATM and DNA-PK knockout cell lines and DNA-PK and ATM specific siRNAs, it was proposed that the S2056 phosphorylation is an autophosphorylation event that occurs in trans while the T2609 phosphorylation is at least in part due to ATM activity (Collis et al., 2005). Further examining the role of autophosphorylation, Neal and Meek (Neal and Meek, 2011; Neal et al., 2011) noted that DNA-PK a relatively abundant protein inhibits the process of $\mathrm{HR}$ in a titratable manner. They also noted that this inhibition is absolutely dependent on its' enzymatic activity and is distinctly modulated by phosphorylation. Through extensive examination of various phosphorylation sites they observed that some sites impact NHEJ negatively while promoting HR. They concluded that failed NHEJ results in increased HR and conversely that in most cases proficient NHEJ inhibits HR. In summary current data suggests that DNA-PK has two important phosphorylation sites: (1) a cluster containing S2056 that is primarily autophosphorylated and (2) a cluster containing sites T2609 and T2647 that are at least in part phosphorylated by ATM. Phosphorylation at these sites is critical as its absence results in retention of DNA-PK foci at damage sites for longer periods. All of these together illustrate the potential for blocking DNA repair by inhibiting DNA-PK phosphorylation. Thus there is vast potential for the use of DNA-PK inhibitors in enhancing DNA damaging chemotherapies.

\section{DNA-PK INHIBITORS}

As such, to date the most successful approach to DNA-PK inhibition has been with small molecules that target the ATP binding site of the kinase domain (Allen et al., 2003). Various investigators using compound libraries have identified a small group of molecules that effectively inhibit DNA-PK kinase activity (Figure 1). However, despite their specificity and potency these molecules have been hampered by poor pharmacokinetic properties.

One of the first identified inhibitors, wortmannin (1), a furanosteroid metabolite of the fungi Penicillium funiculosum, has been used experimentally to inhibit DNA-PK. It is a non-competitive general inhibitor of PI-3 kinases and has an $\mathrm{IC}_{50}$ in the nanomolar range ( $5 \mathrm{nM}$ for PI3K and equipotent for DNA-PK). Its' mode of action involves the irreversible alkylation of lysine 802, an amino acid residing in the active site of DNA-PKcs that is critical for the phosphate transfer reaction. Experimentally wortmannin has
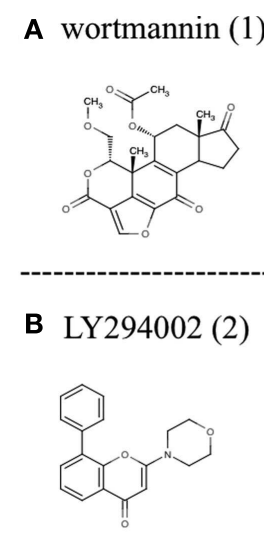

NU7026 (3)

NU7441 (4)

IC86621 (5)<smiles>C=C1C=C([C]2CCCCC2)c2c1ccc1ccccc21</smiles>
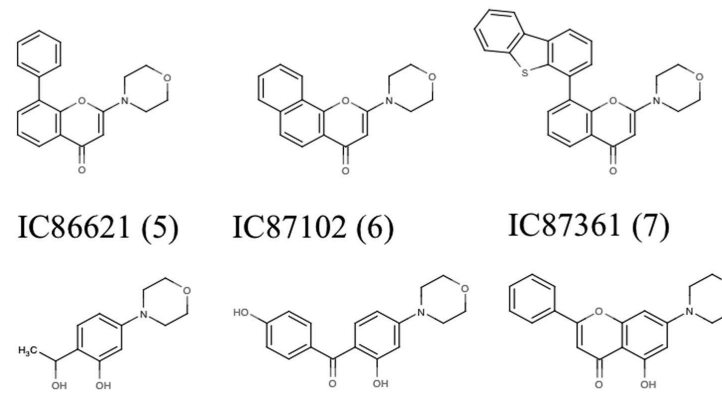

IC87102 (6)

IC87361 (7)
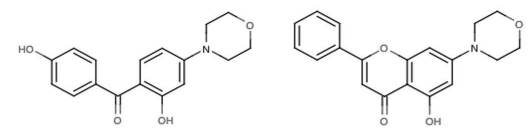

C $\mathrm{OK}-1035(8)$

SU11752 (9)

vanillin (10)<smiles>CCC=Cc1cc(CC=O)cc(-c2ccccc2)c1</smiles>

NK314 (11)

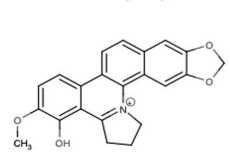

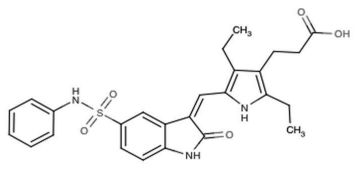

IC486241 (12)
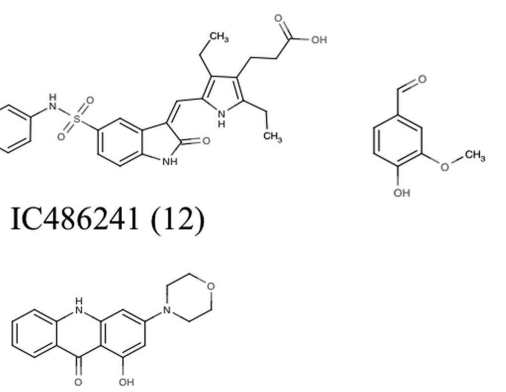

FIGURE 1 | Small molecule inhibitors of DNA-PK: (A) wortmannin, (B) LY294002 derivatives, and (C) other molecules with demonstrated inhibitory activity.

proven to be an effective radiosensitizer with a dose reduction factor (DRF) for IR at 10\% survival between 1.4 and 3. Despite its' experimental efficacy: lack of specificity, poor solubility in aqueous solution, and in vivo toxicity limit clinical application of this compound (Collis et al., 2005).

LY294002 (2), a morpholine derivative of the plant flavonoid quercetin, is also a non-specific DNA-PK inhibitor used in many studies (Vlahos et al., 1994; Sauveur-Michel et al., 2009). It is a competitive inhibitor that binds reversibly to the kinase domain of DNA-PK with an $\mathrm{IC}_{50}$ of $1.4 \mu \mathrm{M}$ producing a DRF at $10 \%$ survival with IR of 1.5-1.8 (Collis et al., 2005). Rapid metabolic clearance $(1 \mathrm{~h})$, in vivo toxicity, and lack of specificity make clinical evaluation unfeasible in humans. However, LY294002 has proven to be a productive lead compound and biochemical modifications have produced a series of compounds with more favorable properties (Fuhrman et al., 2008; Clapham et al., 2011). These modifications using LY294002 as template have improved specificity with regards to DNA-PK inhibition (Ihmaid et al., 2011). 
One such compound NU7026 (3) was over 50 fold more selective for DNA-PK than other PI-3Ks including ATM and ATR. NU7026 had an $\mathrm{IC}_{50}$ of $0.23 \mu \mathrm{M}$ for DNA-PK, $13 \mu \mathrm{M}$ for PI3K, and $>100 \mu \mathrm{M}$ for ATM or ATR (Peddi et al., 2010). Furthermore, this drug potentiated the growth inhibition of a variety of chemotherapeutic agents including: idarubicin, daunorubicin, doxorubicin, etoposide, amsacrine, mitoxantrone but did not potentiate camptothecin or cytosine arabinoside (Willmore et al., 2004). Cell based assays showed a DRF for this drug with IR at $10 \%$ survival of mouse embryonic fibroblasts of 1.5 (Nutley et al., 2005). However, metabolic lability remains problematic as monohydroxylation occurs on position 2 of the morpholino group resulting in opening of the morpholino ring. Thus it is not likely that sufficiently high concentrations of this drug can be obtained in patients over the $4 \mathrm{~h}$ period required to synergize radiation and or chemotherapy. Furthermore, poor solubility in saline solutions limits the use of osmotic mini pumps. It has been suggested that the 2-morpholin-4-yl substituent is required for inhibitory activity. As such attempts have been made to maintain this structure while modifying other parts of the molecule to improve pharmacokinetics. Problematically the core ring structure is not conducive to chemical modifications. As such, Hollick et al. proposed modification of pyran-4-ones and thiopyran-4-ones and obtained suitably potent and selective DNA-PK inhibitors. Although this approach obtained novel compounds screening continues to identify compounds with improved biological and pharmaceutical properties (Hollick et al., 2003).

NU7441 (4) another drug based on the LY294002 backbone with improved potency showed similar promise to NU7026. In cell lines it showed strong inhibition of DNA-PK with an $\mathrm{IC}_{50}$ of $0.3 \mu \mathrm{M}$ for DNA-PK and $7 \mu \mathrm{M}$ for PI3K. In the presence of this compound doxorubicin and IR induced DSBs persisted for prolonged periods and HR activity increased modestly (Rad51 foci; Tavecchio et al., 2012). Zhao and Curtin (Zhao et al., 2006) noted that NU7441 increased the cytotoxicity of IR and etoposide in SW620, LoVo, and V3-YAC colon cancer cells but not in V3 cells indicating that potentiation of DNA damage and cell death was largely due to DNA-PK inhibition. Furthermore, NU7441 substantially delayed the repair of IR and etoposide induced DSBs and appreciably increased G2/M accumulation associated with IR, etoposide and doxorubicin in SW620, and LoVo colon cancer cells. In xenograft colon cancer models NU7441 concentrations in the tumor necessary for chemopotentiation in vitro were maintained for at least $4 \mathrm{~h}$ at non-toxic doses, a significant improvement over NU7026. Additionally, etoposide induced tumor growth delay was increased two-fold without enhancing toxicity. Thus experiments with NU7441 have shown that pharmacokinetics of these inhibitors can be improved but their marginal effects on radiation and chemotherapy have halted further development.

Other compounds based on the LY294002 structure have been tested including IC86621 (5), IC87102 (6), and IC87361 (7). In cell culture these recapitulate the phenotype of DNA-PK defective cell lines including those from SCID mice and directly inhibit the repair of DNA DSBs and enhance the cytotoxicity of physical and chemical agents that induce DSB formation. As with other compounds tested IC86621 showed increased sensitization to IR and decreased repair of spontaneous and IR induced DSBs. Since, these compounds are non-toxic there main therapeutic potential is to enhance the effects of DNA damaging therapies on cancer cells (Kashishian et al., 2003). DNA-PKcs was strongly inhibited and even at concentrations of $100 \mu \mathrm{M}, \mathrm{IC} 86621$ had no activity against the closely related protein kinases ATM, ATR, or FRAP. Additionally, IC87361 showed significant radiosensitization at 6 Gy and significant growth delay in xenograft models. At $10 \mu \mathrm{M}$ concentrations this drug had no intrinsic growth inhibitory properties but significantly potentiated the effect of radiation in ovarian cancer cells. It was observed that at least $4 \mathrm{~h}$ exposure to a $10 \mu \mathrm{M}$ concentration of this drug was required for radiosensitization and that $24 \mathrm{~h}$ of exposure further improved this effect. This time of drug exposure is likely to be important for positive therapeutic effects and illustrates that pharmacokinetic properties such as: rapid clearance from circulation and low bioavailability (Shinohara et al., 2005) are the major barriers to the clinical use of these compounds.

Other compounds based on different chemical structures and found to have inhibitory activity against DNA-PK include OK1035 (8) and SU11752 (9). OK-1035 with an IC 50 for DNA-PK of $100 \mu \mathrm{M}$ is unlikely to be a useful drug in vivo (Take et al., 1996). Similarly, SU11752 was shown to lack the required potency for in vivo studies.

The phenolic aldehyde vanillin (10), derived from some species of vanilla pods, has also been shown to inhibit DNA-PK in cell free extracts. This inhibition was shown to be specific to NHEJ and selective for DNA-PK over ATM and ATR with a DRF of 1.3 at $10 \%$ survival following IR of human ovarian carcinoma cells (Durant and Karran, 2003).

Recently the anticancer agent NK314 (11), possessing activity against topoisomerase II alpha (TII $\alpha$ ), was shown to induce the degradation of DNA-PKcs resulting in impaired DNA DSB repair. In other words NK314 appears to be an inhibitor with dual specificities for TII $\alpha$ and DNA-PKcs with potential activity against adult T-cell leukemia-lymphoma (ATL; Hisatomi et al., 2011). Although the mechanism by which this drug inhibits DNA-PK remains unclear it illustrates the potential for combining DNAPK activity with chemotherapeutics. The combination is in this case all the more effective because the two activities are delivered as a single reagent. This drug is currently in clinical trials for the treatment of ATL illustrating the potential for using DNA-PK inhibitors in the clinic.

Recent studies in our laboratory have explored the efficacy of acridone based compounds as DNA-PK inhibitors (Davidson et al., 2012a,b). These studies sought to measure the degree of synergy induced by the specific small molecule inhibitor of DNA-PK, IC486241 (ICC) (12), with irinotecan (SN38) or oxaliplatin. Significant reductions in the $\mathrm{IC}_{50}$ values of $\mathrm{SN} 38$ were observed at 5 and $10 \mu \mathrm{M}$ of DNA-PK inhibitors. Moreover, at $1-2 \mu \mathrm{M}$ (attainable concentrations with ICC in mice), these DNA$\mathrm{PKcs}$ inhibitors demonstrated synergistic reductions in the $\mathrm{IC}_{50}$ of SN38. Flow cytometric data indicated that SN38 and SN38 in combination with DNA-PKcs inhibitors showed dramatic G2/M arrest at $24 \mathrm{~h}$. Furthermore, reduced phosphorylation of DNA-PKcs and increased DNA damage were observed at this time point with SN38 in combination with DNA-PKcs inhibitors as compared to cells treated with SN38 alone. SN38 alone and in the presence of ICC increased nuclear Rad51 protein levels. Furthermore, inhibition of DNA-PKcs increased HRR suggesting that NHEJ is a 
negative regulator of HRR. This observation was corroborated by Neal et al. (2011), who showed that DNA-PK enzymatic activity could inhibit HRR in a titratable fashion. In addition these authors also showed that some phosphorylations of DNA-PK had the opposite effect, that is to say, inhibition of NHEJ and promotion of HRR. Of interest, Amrein et al. (2011) observed a similar relationship between the HRR and NHEJ pathways in chronic lymphocytic leukemia (CLL) lymphocytes treated with chlorambucil (CLB). Imatinib and Nilotinib, two potent inhibitors of the nonreceptor tyrosine kinase $\mathrm{c}-\mathrm{abl}$, caused a reduction in HRR and a corresponding increase in DNA-PK (NHEJ) activity. Together these observations suggest that DNA-PK has a significant function in choosing the pathway of DNA repair. A similar series of experiments showed the degree to which ICC synergizes the cytotoxicity of DNA damaging agents in three genetically diverse breast cancer cell lines. In this study, improved cytotoxicity and significant synergy were observed with both anticancer agents in the presence of non-toxic concentrations of ICC. Moreover, ICC decreased doxorubicin-induced DNA-PKcs autophosphorylation on Ser2056 and increased doxorubicin-induced DNA fragmentation. Thus, the novel DNA-PKcs inhibitor, ICC, synergistically sensitized 3 breast cancer cell lines to doxorubicin and cisplatin. Enhanced efficacy of doxorubicin was achieved by inhibiting NHEJ resulting in increased accumulation of DNA damage. Pharmacokinetic studies of these compounds in mice showed maximum serum concentrations of $4.6 \mu \mathrm{M} 1 \mathrm{~min}$ post intravenous injection and terminal serum half-lives of $23.8 \mathrm{~min}$ (personal communication, Luipold pharmaceuticals). Thus although this drug shows promising synergy with chemotherapeutics in cell culture, future use will depend on developing compounds with improved solubility and serum half-life.

\section{NUCLEOTIDE AND ANTIBODY INHIBITORS}

Although the majority of studies have focused on the use of small organic compounds to inhibit DNA-PK, a few have focused on nucleotide and antibody based inhibitors and shown that these may also have clinical efficacy in the inhibition of DNA-PK. In fact due to the biological nature of these molecules they may overcome the two primary obstacles faced by small organic compounds known to inhibit DNA-PK, namely: poor solubility's and short serum half-lives. GRN163L (Imetelstat; GRN), a 13-mer oligonucleotide developed as a direct inhibitor of the telomerase active site illustrates how nucleotides can influence DNA-PK function. In a recent study in CLL lymphocytes this molecule was shown to inhibit DNA-PK phosphorylation and increase H2AX phosphorylation in response to treatment with the nucleotide analog fludarabine (flu; Shawi et al., 2011). The authors observed that

\section{REFERENCES}

Allen, C., Halbrook, J., and Nickoloff, J. A. (2003). Interactive competition between homologous recombination and non-homologous end joining. Mol. Cancer Res. 1, 913-920.

Amrein, L., Davidson, D., Shawi, M., Petruccelli, L. A., Miller, W. H. Jr., Aloyz, R., et al. (2011). Dual inhibition of the homologous

activity was correlated with low expression levels of the DNA-PK subunit Ku80 and that GRN inhibited DNA-PK phosphorylation at a level equivalent to NU7026. Together these observations indicate that GRN in addition to inhibiting telomerase activity enhances chemotherapy by inhibiting DNA-PK activity and repair of DNA damage. It would be of significant interest to know if GRN synergizes the killing of other cancer types in the presence of a variety of DNA damaging therapies.

In addition to oligonucleotides, antibody based inhibitors may also have a role to play in DNA-PK inhibition. Antibodies, although effective in the inhibition of cell surface targets (Scott, 2012) are typically ineffective against intracellular targets because of their inability to penetrate the plasma membrane (Cardinale and Biocca, 2008). However, recent work in which the DNA-PK specific single-chain antibody variable region fragment ScFv 18-2 was linked to folate via a scissile disulfide linker showed efficacy in delivering the antibody fragment to cell nuclei via folate mediated endocytosis. This product resulted in decreased DNA-PK phosphorylation, increased $\mathrm{H} 2 \mathrm{AX}$ phosphorylation, and radiosensitization (Xiong et al., 2011). This work is of interested for a number of reasons. Firstly, it illustrates the potential of antibody based therapies that target intracellular molecules such as DNAPK and secondly it illustrates the efficacy of combi-molecules composed of a cell surface receptor substrate and a chemotherapeutic agent. The receptor substrate as in this case can facilitate transit of the plasma and nuclear membranes but may also be used to target drugs to cancers over-expressing specific surface receptors.

In conclusion DNA-PK inhibition using small molecule inhibitors holds promise for improving cancer therapy. However, molecules currently known to specifically inhibit DNA-PK are limited by poor pharmacokinetics: metabolic lability leads to a short serum half-life. Future improvement in DNA-PK inhibition will likely be achieved by designing new molecules based on the recently reported crystallographic structure of DNA-PK (Sibanda et al., 2010; Ihmaid et al., 2012). Computer based drug design will not only assist in identifying novel functional moieties to replace the metabolically labile morpholino group but will also facilitate the design of molecules to target the DNA-PKcs/Ku80 interface or one of the autophosphorylation sites. Of significant interest novel approaches using DNA-PK specific antibodies or oligonucleotides may also be of great value as they may overcome the fundamental weaknesses of currently available small molecule inhibitors of DNA-PK, namely: poor solubility and short serum half-life. To date the latter have only been tested against DNA-PK in vitro, future work will demonstrate if these approaches have significant in vivo efficacy.

cell cycle progression. BMC Mol. Biol. 11:18. doi:10.1186/1471-219911-18

Azad, A., Jackson, S., Cullinane, C., Natoli, A., Neilsen, P. M., Callen, D. F., et al. (2011). Inhibition of DNA-dependent protein kinase induces accelerated senescence in irradiated human cancer cells. Mol. Cancer Res. 9, 1696-1707.
Bennardo, N., Cheng, A., Huang, N., and Stark, J. M. (2008). Alternative-nhej is a mechanistically distinct pathway of mammalian chromosome break repair. PLoS Genet. 4:e1000110. doi:10.1371/journal.pgen.1000110

Burma, S., Chen, B. P. C., and Chen, D. J. (2006). Role of non-homologous end joining (NHEJ) in maintaining genomic integrity. DNA Repair (Amst.) 5, 1042-1048. 
Callen, E., Jankovic, M., Wong, N., Zha, S., Chen, H.-T., Difilippantonio, S., et al. (2009). Essential role for DNA-PKcs in DNA double-strand break repair and apoptosis in ATMdeficient lymphocytes. Mol. Cell 34, 285-297.

Cardinale, A., and Biocca, S. (2008). The potential of intracellular antibodies for therapeutic targeting of protein-misfolding diseases. Trends. Mol. Med. 14, 373-380.

Cardoso, F., Bedard, P. L., Winer, E. P., Pagani, O., Senkus-Konefka, E., Fallowfield, L. J., et al. (2009). International guidelines for management of metastatic breast cancer: combination vs sequential single-agent chemotherapy. J. Natl. Cancer Inst. 101, 1174-1181.

Chen, B. P. C., Chan, D. W., Kabayashi, J., Burma, S., Asaithamby, A., Morotomi-Yano, K., et al. (2005). Cell cycle dependence of DNAdependent protein kinase phosphorylation in response to DNA double strand breaks. J. Biol. Chem. 280, 14709-14715.

Clapham, K. M., Bardos, J., Finlay, M. R. V., Golding, B. T., Griffen, E. J., Griffin, R. J., et al. (2011). DNA-dependent protein kinase (DNA-PK) inhibitors: Structure-activity relationships for o-alkoxyphenylchromen-4one probes of the ATP-binding domain. Bioorg. Med. Chem. Lett. 21, 966-970.

Collis, S. J., DeWeese, T. L., Jeggo, P. A., and Parker, A. R. (2005). The life and death of DNA-PK. Oncogene 24, 949-961.

Davidson, D., Coulombe, Y., MartinezMarignac, V., Amrein, L., Grenier, J., Hodkinson, K., et al. (2012a). Irinotecan and DNA-PKcs inhibitors synergize in killing of colon cancer cells. Invest. New Drugs 30, 1248-1256.

Davidson, D., Grenier, J., MartinezMarignac, V., Amrein, L., Shawi, M., Tokars, M., et al. (2012b). Effects of the novel DNA dependent protein kinase inhibitor, IC486241, on the DNA damage response to doxorubicin and cisplatin in breast cancer cells. Invest. New Drugs 30, 1736-1742.

Davis, A. J., So, S., and Chen, D. J. (2010). Dynamics of the PI3K-like protein kinase members ATM and DNA-PKcs at DNA double strand breaks. Cell Cycle 9, 2527-2534.

Dobbs, T. A., Tainer, J. A., and Lees-Miller, S. P. (2010). A structural model for regulation of nhej by DNA-PKcs autophosphorylation. DNA Repair (Amst.) 9, 1307-1314.
Durant, S., and Karran, P. (2003). Vanillins, a novel family of DNAPK inhibitors. Nucleic Acids Res. 31, 5501-5512.

Durocher, D., and Jackson, P. J. (2001). DNA-PK, ATM and ATR as sensors of DNA damage: variations on a theme? Curr. Opin. Cell Biol. 13, 225-231.

Fuhrman, C. B., Kilgore, J., LaCoursiere, Y. D., Lee, C. M., Milash, B. A., Soisson, A. P., et al. (2008). Radiosensitization of cervical cancer cells via double-strand DNA break repair inhibition. Gynecol. Oncol. 110, 93-98.

Hisatomi, T., Sueoka-Aragane, N., Sato, A., Tomimasu, R., Ide, M., Kurimasa, A., et al. (2011). NK314 potentiates anti-tumor activity with adult t-cell leukemia-lymphoma cells by inhibition of dual targets on topoisomerase II and DNA-dependent protein kinase. Blood 117, 3575-3584.

Hollick, J. J., Golding, B. T., Hardcastle, I. R., Martin, N., Richardson, C., Rigoreau, L. J. M., et al. (2003). 2,6-disubstituted pyran-4one and thiopyran-4-one inhibitors of DNA-dependent protein kinase (DNA-PK). Bioorg. Med. Chem. Lett. 13, 3083-3086.

Ihmaid, S., Al-Rawi, J., Bradley, C. Angove, M. J., Robertson, M. N., and Clark, R. L. (2011). Synthesis, structural elucidation, DNA-PK inhibition, homology modelling and anti-platelet activity of morpholinosubstituted-1,3-naphth-oxazines. Bioorg. Med. Chem. 19, 3983-3994.

Ihmaid, S. K., Al-Rawi, J. M., Bradley, C. J., Angove, M. J., and Robertson, M. N. (2012). Synthesis, DNA-PK inhibition, anti-platelet activity studies of 2(N-substituted-3-aminopyridine)substituted-1,3-benzoxazines and DNA-PK and PI3K inhibition, homology modelling studies of 2-morpholino-(7,8-di and 8substituted)-1,3-benzoxazines. Eur. J. Med. Chem. 5, 85-101.

Johnson, R. D., and Jasin, M. (2001). Double-strand-break-induced homologous recombination in mammalian cells. Biochem. Soc. Trans. 29, 196-201.

Kao, J., Rosenstein, B. S., Peters, S., Milano, M. T., and Kron, S. J. (2005). Cellular response to DNA damage. Ann. N. Y. Acad. Sci. 1066, 243-258.

Kashishian, A., Douangpanya, H., Clark, D., Schlachter, S. T., Eary, C. T., Schiro, J. G., et al. (2003). DNAdependent protein kinase inhibitors as drug candidates for the treatment of cancer. Mol. Cancer Ther. 2, 1257-1264.
Koike, M., Sugasawa, J., Yasuda, M. and Koike, A. (2008). Tissue-specific DNA-PK-dependent H2AX phosphorylation and $\mathrm{y}-\mathrm{H} 2 \mathrm{AX}$ elimination after $\mathrm{x}$-irradiation in vivo. Biochem. Biophys. Res. Commun. 376, 52-55.

Kong, X., Shen, Y., Jiang, N., Fei, X., and Mi, J. (2011). Emerging roles of DNA-PK besides DNA repair. Cell. Signal. 23, 1273-1280.

Kuo, C. C., Liu, J. F., and Chang, J. Y. (2006). DNA repair enzyme, O6-methylguanine DNA methyltransferase, modulates cytotoxicity of camptothecin-derived topoisomerase I inhibitors. J. Pharmacol. Exp. Ther. 316, 946-954.

Lieber, M. R. (2008). The mechanism of human nonhomologous DNA end joining. J. Biol. Chem. 283, 1-5.

Lieber, M. R., Yu, K., and Raghavan, S. C. (2006). Roles of nonhomologous DNA end joining, $\mathrm{v}(\mathrm{d}) \mathrm{j}$ recombination, and class switch recombination in chromosomal translocations. DNA Repair (Amst.) 5, 1234-1245.

Ma, Y., Pannicke, U., Schwarz, K., and Lieber, M. R. (2002). Hairpin opening and overhang processing by an artemis/DNA-dependent protein kinase complex in nonhomologous end joining and $\mathrm{v}(\mathrm{d}) \mathrm{j}$ recombination. Cell 108, 781-794.

Mansour, W. Y., Schumacher, S., Rosskopf, R., Rhein, T., SchmidtPetersen, F., Gatzemeier, F., et al. (2008). Hierarchy of nonhomologous end-joining, singlestrand annealing and gene conversion at site-directed DNA doublestrand breaks. Nucleic Acids Res. 36 4088-4098.

Morris, E. P., Rivera-Calzada, A., da Fonseca, P. C. A., Llorca, O., Pearl, L. H., and Spagnolo, L. (2011). Evidence for a remodelling of DNAPK upon autophosphorylation from electron microscopy studies. Nucleic Acids Res. 39, 5757-5767.

Mukherjee, B., Kessinger, C., Kobayashi, J., Chen, B., Chen, D., Chatterjee, A., et al. (2006). DNA-PK phosphorylates histone H2AX during apoptotic DNA fragmentation in mammalian cells. DNA Repair (Amst.) 5 , 575-590.

Neal, J. A., Dang, V., Douglas, P., Wold, M. S., Lees-Miller, S. P., and Meek, K. (2011). Inhibition of homologous recombination by DNA-dependent protein kinase requires kinase activity, is titratable, and is modulated by autophosphorylation. Mol. Cell. Biol. 31, 1719-1733.

Neal, J. A., and Meek, K. (2011). Choosing the right path: does DNA-PK help make the decision? Mutat. Res. 711, 73-86.

Nutley, B. P., Smith, N. F., Hayes, A., Kelland, L. R., Brunton, L., Golding, B. T., et al. (2005). Preclinical pharmacokinetics and metabolism of a novel prototype DNA-PK inhibitor NU7026. Br. J. Cancer 93 1011-1018.

Peddi, P., Loftin, C. W., Dickey, J. S., Hair, J. M., Burns, K. J., Aziz, K., et al. (2010). DNA-PKcs deficiency leads to persistence of oxidatively induced clustered DNA lesions in human tumor cells. Free Radic. Biol. Med. 48, 1435-1443.

Raftery, L., and Goldberg, R. M. (2010). Optimal delivery of cytotoxic chemotherapy for colon cancer. Cancer J. 16, 214-219.

Reitsema, T., Klokov, D., Banath, J., and Olive, P. (2005). DNA-PK is responsible for enhanced phosphorylation of histone H2AX under hypertonic conditions. DNA Repair (Amst.) 4, 1172-1181.

Rudolf, E., Rudolf, K., and Cervinka, M. (2011). Camptothecin induces p53dependent and -independent apoptogenic signaling in melanoma cells. Apoptosis 16, 1165-1176.

Sauveur-Michel, M., Stauffer, F., Schnell, C., and Garcia-Echeverria, C. (2009). PI3K inhibitors for cancer treatment: where do we stand? Biochem. Soc. Trans. 37, 265-272.

Scott, A. M. (2012). Antibody therapy of cancer. Nat. Rev. Cancer 12, 278-287.

Seymour, M. T., Maughan, T. S., Ledermann, J. A., Topham, C., James, R. Gwyther, S. J., et al. (2007). Different strategies of sequential and combination chemotherapy for patients with poor prognosis advanced colorectal cancer (mrc focus): a randomised controlled trial. Lancet 370 , 143.

Shawi, M., Amrein, L., Gryaznov, S. M., Autexier, C., and Aloyz, R. (2011). Telomerase inhibition affects fludaribine sensitivity in chronic lymphocytic leukemia lymphocytes. Eur. J. Cancer 47, S640-S641.

Shinohara, E. T., Geng, L., Tan, J., Chen, H., Shir, Y., Edwards, E., et al. (2005). DNA-dependent protein kinase is a molecular target for the development of noncytotoxic radiationsensitizing drugs. Cancer Res. 65, 4987-4992.

Sibanda, B. L., Chirgadze, D. Y., and Blundell, T. L. (2010). Crystal structure of DNA-PKcs reveals a large open-ring cradle comprised of heat repeats. Nature 463, 118-121.

Soubeyrand, S., Pope, L., Pakuts, B., and Hache, J. G. (2003). Threonines 
2638/2647 in DNA-PK are essential for cellular resistance to ionizing radiation. Cancer Res. 63, 1198-1201.

Stiff, T., O’Driscoll, M., Rief, N., Iwabuchi, K., Lobrich, M., and Jeggo, P. (2004). ATM and DNAPK function redundantly to phosphorylate $\mathrm{H} 2 \mathrm{AX}$ after exposure to ionizing radiation. Cancer Res. 64, 2390-2396.

Stronack, E. A., Chen, M., Maginn, E. N., Agarwai, R., Mills, G. B., Harpreet, W., et al. (2011). DNA-PK mediates AKT activation and apoptosis inhibition in clinically acquired platinum resistance. Neoplasia 13, 1069-1080.

Sung, P., and Klein, H. (2006). Mechanism of homologous recombination: mediators and helicases take on regulatory functions. Nat. Rev. Mol. Cell Biol. 7, 739-750.

Take, Y., Kumano, M., Hirobumi, T., Nishimura, S., and Okuyama, A. (1996). DNA-dependent protein kinase inhibitor (OK-1035) suppresses p21 expression in HCT-116 cells containing wildtype p53 induced by adriamycin. Biochem. Biophys. Res. Commun. 221, 207-212.

Tavecchio, M., Munck, J., Cano, C., Newell, D., and Curtin, N. (2012). Further characterisation of the cellular activity of the DNA-pk inhibitor,
NU7441, reveals potential cross-talk with homologous recombination. Cancer Chemother. Pharmacol. 69, 155-164.

Thompson, L. H. (2012). Recognition, signaling, and repair of DNA double-strand breaks produced by ionizing radiation in mammalian cells: the molecular choreography. Mutat. Res. 751, 158-246.

Tol, J., Koopman, M., Cats, A., Rodenburg, C. J., Creemers, G. J. M., Schrama, J. G., et al. (2009). Chemotherapy, bevacizumab, and cetuximab in metastatic colorectal cancer. N. Engl. J. Med. 360, 563-572.

Uematsu, N., Weterings, E., MorotomiYano, K., Jakob, B., Taucher-Scholz, G., Mari, P.-O., et al. (2007). Autophosphorylation of DNA-PKcs regulates its dynamics at DNA double-strand breaks. J. Cell Biol. 177, 219-229.

Vlahos, C. J., Matter, W. F., Hui, K. Y., and Brown, R. F. (1994). A specific inhibitor of phosphatidylinositol 3-kinase, 2-(4-morpholinyl)-8phenyl-4h-1-benzopyran-4-one (ly294002). J. Biol. Chem. 269, 5241-5248.

Wang, H., Wang, M., Bocker, W., and Iliakis, G. (2005). Complex H2AX phosphorylation patterns by multiple kinases including ATM and DNA-PK in human cells exposed to ionizing radiation and treated with kinase inhibitors. J. Cell. Physiol. 202, 492-502.

Wei, S., Xiong, M., Zhan, D.-Q. Liang, B.-Y., Wang, Y.-Y., Gutmann, D. H., et al. (2012). Ku80 functions as a tumor suppressor in hepatocellular carcinoma by inducing s-phase arrest through a p53dependent pathway. Carcinogenesis 33, 538-547.

Willmore, E., de Caux, S., Sunter, N. J. Tilby, M. J., Jackson, G. H., Austin, C. A., et al. (2004). A novel DNAdependent protein kinase inhibitor, NU7026, potentiates the cytotoxicity of topoisomerase II poisons used in the treatment of leukemia. Blood 103, 4659-4665

Xiong, H., Lee, R. J., Haura, E. B. Edwards, J. G., Dynan, W. S., and Shuyi, L. (2011). Intranuclear delivery of a novel antibody-derived radiosensitizer targeting the DNAdependent protein kinase catalytic subunit. Int. J. Radiat. Oncol. Biol. Phys. 83, 1023-1030.

Yang, C., Betti, C., Singh, S., Toor, A., and Vaughan, A. (2009). Impaired NHEJ function in multiple myeloma. Mutat. Res. 660, 66-73.

Yang, J., Yingnian, Y., Hamrick, H. E., and Duerksen-Hughes, J. (2003). ATM, ATR and DNA-PK: initiators of the cellular genotoxic stress responses. Carcinogenesis 24, 1571-1580.
Zhao, Y., Thomas, H. D., Batey, M. A., Cowell, I. G., Richardson, C. J. Griffin, R. J., et al. (2006). Preclinical evaluation of a potent novel DNA-dependent protein kinase inhibitor NU7441. Cancer Res. 66 , 5354-5362.

Conflict of Interest Statement: The authors declare that the research was conducted in the absence of any commercial or financial relationships that could be construed as a potential conflict of interest.

Received: 24 October 2012; paper pending published: 06 December 2012; accepted: 08 January 2013; published online: 31 January 2013.

Citation: Davidson D, Amrein L, Panasci $L$ and Aloyz $R$ (2013) Small molecules, inhibitors of DNA-PK, targeting DNA repair, and beyond. Front. Pharmacol. 4:5. doi: 10.3389/fphar.2013.00005

This article was submitted to Frontiers in Pharmacology of Anti-Cancer Drugs, a specialty of Frontiers in Pharmacology. Copyright (C) 2013 Davidson, Amrein, Panasci and Aloyz. This is an openaccess article distributed under the terms of the Creative Commons Attribution License, which permits use, distribution and reproduction in other forums, provided the original authors and source are credited and subject to any copyright notices concerning any third-party graphics etc. 